\title{
Understanding the growth of massive galaxies via stellar populations
}

\author{
Ignacio Ferreras \\ MSSL, University College London, Holmbury St Mary, Dorking, Surrey, UK \\ email: ferreras@star.ucl.ac.uk
}

\begin{abstract}
The formation and evolution of massive galaxies represent one of the most intriguing open problems in astrophysics. Their underlying stellar populations encode valuable information about their past history. Detailed spectroscopic observations allow us to constrain the star formation histories, revealing a complicated mixture of a strong, early formation process, followed by passive evolution in the cores, along with an extended assembly of the outer regions via minor mergers. In this contributed talk, some recent results are presented from the analysis of samples of massive galaxies both at $z \sim 0$ and moderate redshift.
\end{abstract}

\section{Introduction}

Massive galaxies constitute one of the best testbeds of our understanding of galaxy formation. Due to the inherent bottom-up hierarchical growth of structure within the best-fit cosmological model (i.e. $\Lambda \mathrm{CDM}$ ), the most massive structures assemble later. This result is in tension with the overall old stellar populations found in massive galaxies. However, a suitable understanding of the baryonic processes leading from gas infall to star formation and feedback would be able to reconcile the observations with the expected growth of structure within a $\Lambda$ CDM cosmology. Therefore, massive galaxies can be used to set strong constraints on models of galaxy formation.

The standard process of galaxy growth by the cooling of shock-heated gas after the collapse of a structure (White \& Rees 1978), which has been adopted by most of the numerical simulations of galaxy formation to date, has been revised in the past few years, by the discovery of a new growth mechanism, based on the inflow of cold gas along filaments (see, e.g. Dekel \& Birnboim 2006). Cold accretion can fuel early, efficient stages of star formation, that could account, in part, for the formation of the most massive galaxies at early times $(z>3)$. In addition, massive galaxies have been found to evolve in size during a later phase $(z<3)$; with a change up to a factor of 4 between $z=1.5$ and today (see, e.g. Trujillo et al. 2007). Numerical simulations suggest a two-phase formation scenario, whereby a first period of formation, driven by cold accretion, creates a massive "core" of metal-rich stars, followed by a prolongued process of minor merging, that mainly populate the regions external to this core, with metal-poorer stars (Naab et al. 2009; Oser et al. 2010).

We present below a number of cases where the analysis of the underlying stellar populations allow us to constrain the formation mechanism of massive galaxies over cosmic time. This contributed talk briefly presents work done in collaboration with a long list of cherished colleagues.

\section{CASE A: Massive galaxies at $z \sim 0$}

The field of spectroscopic dating of unresolved stellar populations has come of age over the past decade, with the large volume of high quality data provided by the Sloan 
Digital Sky Survey (SDSS, York et al. 2000), and the availability of population synthesis models with comparable spectral resolution (Bruzual \& Charlot 2003). The spectra of massive galaxies $\left(M_{s}>10^{11} M_{\odot}\right)$ at $z \sim 0$, which mainly correspond to an early-type morphology, are dominated by old, metal-rich populations (see, e.g., Thomas et al. 2005). Detailed analyses based on spectral fitting reveal an early, intense, short-lived process of star formation, with $80 \%$ of the stellar mass already formed within the first $3 \mathrm{Gyr}$ (de la Rosa et al. 2011). In addition, we find an abrupt transition in the star formation history of galaxies with stellar mass below $\gtrsim 3 \times 10^{10} \mathrm{M}_{\odot}$, suggesting a different channel of formation (Trevisan et al. 2012).

These studies focus on the central regions of the galaxies (the fiber-fed SDSS spectra only probe an aperture of $3 \operatorname{arcsec}$ ). It is more challenging to explore radial gradients of stellar populations with spectroscopy out to several effective radii. Some studies on a small set of targets find very small age radial gradients and a significant change in the metallicity gradient with respect to galaxy mass (Sánchez-Blázquez et al. 2007, Spolaor et al. 2009). A photometric optical-NIR analysis of the radial profiles of the populations in a large sample of massive ETGs from SDSS reveal a metallicity-dominated trend (decreasing outwards), with homogeneously old populations out to an effective radius (La Barbera et al. 2012), and a steepening of the metallicity gradient, along with a trend towards older populations at distances $4-8 \mathrm{R}_{e}$ (La Barbera et al. 2012). These results are consistent with numerical simulations, where the infall of small structures, formed early, would populate the outskirts of massive galaxies (Lackner et al. 2012).

\section{CASE B: Massive, early-type galaxies at moderate redshift}

At higher redshifts, massive galaxies span a wider range of morphologies in constrast to the ETG-dominated population at low redshift. We focus here on the $z \sim 0.7$ Universe, where ETGs constitute a fraction $\gtrsim 60 \%$ of all massive galaxies (Buitrago et al. 2013). So far, the best spectra for the analysis of the stellar populations in this range has been taken by the slitless grisms of ACS and WFC3, instruments onboard HST. The superb flux calibration of these data allows for a robust constraint of the ages and metallicities of galaxies at moderate redshift (Ferreras et al. 2009a), confirming the quiescent star formation histories obtained in the low-redshift sample. Photometric analyses covering a much wider range in redshift give a similar picture, with the bulk of the stellar populations of massive galaxies forming at redshifts $z_{F} \gtrsim 3$ (see, e.g., Pérez-González et al. 2005). The analysis of radial gradients in the properties of the underlying populations at $z \sim 0.7$ is currently limited to photometric data, and consistently show a nearly-flat colour gradient out to an effective radius, with the main trend driven by metallicity (Ferreras et al. 2005). The presence of younger populations in spheroidal galaxies at those redshifts is only found in the form of blue cores, at stellar masses $\lesssim 10^{10} \mathrm{M}_{\odot}$ (Pasquali et al. 2006, Ferreras et al. 2009 b).

\section{CASE C: Size evolution and stellar populations}

In order to understand the growth in size of massive galaxies between $z \sim 2$ and 0, we compared the SDSS-based samples presented in cases A and B above, using the same methodology to extract their star formation histories (Trujillo et al. 2011). The analysis found no significant difference in the population ages on the mass-size plane, ruling out growth scenarios where a significant amount of star formation is expected, as in the puffing-up mechanism of Fan et al. (2008), or the emergence hypothesis of van der Wel et al. (2008), where a contribution in the "emergent" systems created from 

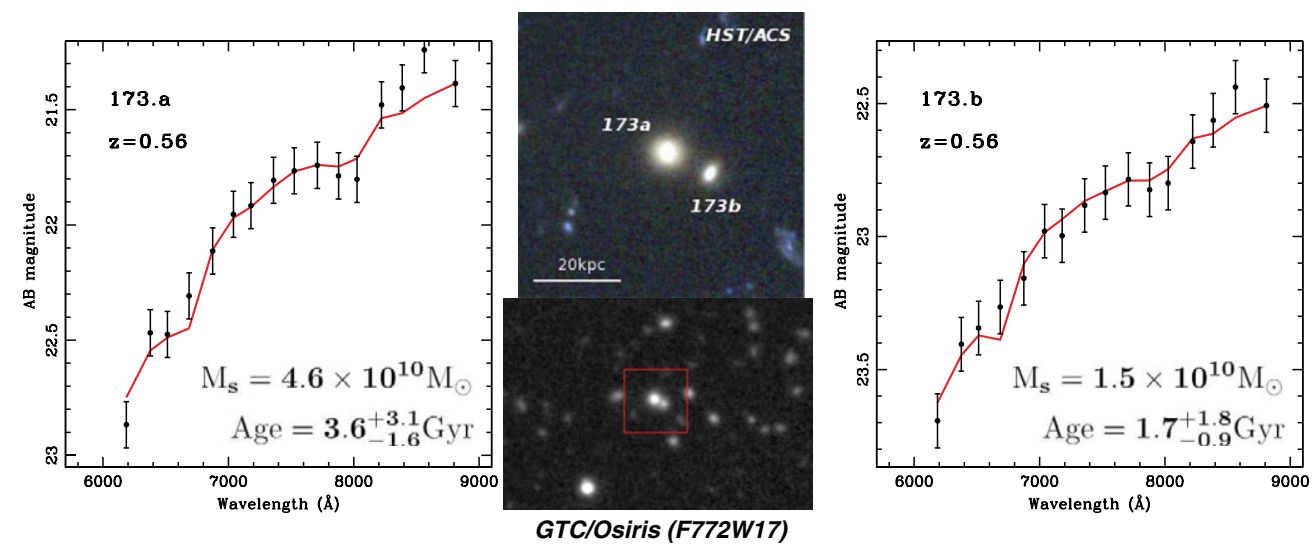

Figure 1. An example of the analysis of stellar populations in close pairs involving a massive galaxy, with data from the SHARDS collaboration (Pérez-González et al. 2013). ACS/HST, and OSIRIS/GTC images are shown in the central top and bottom panels, respectively. The left/right diagrams correspond to the data (points) and best SSP model (line) for the centreal (left) and satellite galaxy, respectively (Ferreras et al., in preparation).

late-type galaxies to the $z \sim 0$ census of massive galaxies would have shown up as a measurable fraction of young stars in the analysis of de la Rosa et al. (2011). Hence, the most plausible scenario involves dry minor mergers over this redshift range (Naab et al. 2009). A simple analysis of the evolution of galaxies on the mass-size plane shows that a relatively small number of minor mergers is needed to account for the growth between $z \sim 1$ and 0 (Trujillo et al. 2011), consistent with the fraction of observed satellites around massive spheroidal galaxies (Mármol-Queraltó et al. 2012). This study was followed up by an analysis of the stellar populations using broadband photometry, finding similar stellar ages between the populations of the central (massive) and satellite galaxies at high redshift, with a trend towards an age difference $t_{C}-t_{S} \gtrsim 1.5 \mathrm{Gyr}$ at lower redshifts (Mármol-Queraltó et al. 2013).

We are continuing this project of characterization of the populations of (massive) centrals and satellites at $z \lesssim 1$ with the SHARDS survey, involving deep photometry of the GOODS North $130 \operatorname{arcmin}^{2}$ field with 24 contiguous medium-width filters in the spectral range 5000-9500Å (Pérez-González et al. 2013). The dataset effectively provide $R \sim 50$ spectra down to $\mathrm{AB}=26.5 \mathrm{mag}(3 \sigma)$, enabling us to achieve an accurate determination of the redshifts (essential to select close pairs), and to probe in detail the stellar populations of the companions of massive galaxies at $z \lesssim 1$. Figure 1 gives an example for a close pair at $z=0.56$, with a simple comparison with SSP models from Bruzual \& Charlot (2003).

\section{CASE D: A different formation channel at early times?}

Observational evidence, therefore, points towards a two-stage formation process in massive ETGs, involving an initial phase of rapid, early star formation between $z_{F} \sim$ $5-10$ and 2 , followed by a general quiescent behaviour along with stellar mass growth of mostly gas-poor minor mergers. The physical conditions of star formation during the rapid early phase must have been significantly different from the bulk of star forming galaxies at lower redshifts. Detailed spectroscopic analyses of the populations of massive galaxies at $z \gtrsim 2$ (see, e.g. van Dokkum \& Brammer 2010, Ferreras et al. 2012, Toft et al. 2012) will enable us to quantify the relative importance of mergers and cold accretion to the process. 
Preliminary studies of the IMF under those conditions suggest a trend towards a bottom-heavy IMF in strong star-bursting systems (Hopkins 2012), similar to the observed trend in gravity-sensitive line strengths that reveal an excess of low-mass stars in the most massive galaxies (van Dokkum \& Conroy 2010, Spiniello et al. 2012, Ferreras et al. 2013). Although those studies still need to improve, to be able to robustly constrain the shape of the low-mass end of the IMF, independent work based on dynamical modelling, or strong gravitational lensing seem to suggest a similar scenario (Auger et al. 2010, Thomas et al. 2011, Cappellari et al. 2012).

\section{References}

Auger, M. W., et al. 2010, ApJ Lett., 721, L163

Bruzual, G. \& Charlot, S. 2003, MNRAS, 344, 1000

Buitrago, F., et al. 2013, MNRAS, 428, 1460

Cappellari, M., et al. 2012, Nature, 484, 485

de la Rosa, I. G., et al. 2011, MNRAS Lett., 418, L74

Dekel, A. \& Birnboim, Y. 2006, MNRAS, 368, 2

Fan, L., Lapi, A., De Zotti, G., \& Danese, L. 2008, ApJ Lett, 689, L101

Ferreras, I., et al. 2005, ApJ, 635, 243

-. 2009a, ApJ, 706, 158

-. 2009b, MNRAS, 396, 1573

-. 2012, AJ, 144, 47

-. 2013, MNRAS Lett., 429, L15

Hopkins, P. 2012, MNRAS, submitted (arXiv:1204.2835)

La Barbera, F., et al. 2011, ApJL, 740, L41

-. 2012, MNRAS, 426, 2300

Lackner, C., et al. 2012, MNRAS, 425, 641

Mármol-Queraltó, E., et al. 2012, MNRAS, 422, 2187

—. 2013, MNRAS, 429, 792

Naab, T., Johansson, P. H., \& Ostriker, J. P. 2009, ApJ Lett., 699, L178

Oser, L., et al. 2010, ApJ, 725, 2312

Pasquali, A., et al. 2006, ApJ, 636, 115

Pérez-González, P., et al. 2005, ApJ, 630, 82

—. 2013, ApJ, 762, 46

Sánchez-Blázquez, P., et al. 2007, MNRAS, 377, 759

Spiniello, C., et al. 2012, ApJ Lett, 753, L32

Spolaor, M., et al. 2009, ApJ Lett., 691, L138

Thomas, D., et al. 2005, ApJ,621, 673

Thomas, J., et al. 2011, MNRAS, 415, 545

Toft, S., et al. 2012, ApJ, 754, 3

Trevisan, M., et al. 2012, ApJ Lett., 752, L27

Trujillo, I., et al. 2007, MNRAS, 382, 109

Trujillo, I., Ferreras, I., \& de la Rosa, I. G. 2011, MNRAS, 415, 3903

Valentinuzzi, T., et al. 2010, ApJ, 712, 226

van Dokkum, P., et al. 2008, ApJ Lett., 677, L5

van Dokkum, P. \& Brammer, G. 2010, Nature, 468, 940

van Dokkum, P. \& Conroy, C. 2010, Nature, 468, 940

Vazdekis, A., et al. 2012, MNRAS, 424, 157

White S. D. M., Rees, M. J. 1978, MNRAS, 183, 341

York, D., et al. 2000, AJ, 120, 1579 